\title{
Male predominance in ketosis-prone diabetes mellitus (Review)
}

\author{
XIAOHAO WANG and HUIWEN TAN \\ Department of Endocrinology and Metabolism, West China Hospital of Sichuan University, \\ Chengdu, Sichuan 610041, P.R. China
}

Received February 27, 2015; Accepted April 22, 2015

DOI: $10.3892 /$ br.2015.461

\begin{abstract}
The incidence of ketosis-prone diabetes mellitus (KPDM) shows a higher prevalence in men. The clear male predominance of this syndrome and its underlying pathogenesis mechanisms are unclear. KPDM, once described as atypical diabetes mellitus, idiopathetic type 1 diabetes (type 1B diabetes) and flatbush diabetes, is an uncommon form of diabetes characterized by severe reversible insulin deficiency. KPDM was first described and mostly observed in males of African-American descent and recently in Asian populations, including Japanese and Chinese. Patients with KPDM often present acutely with diabetic ketoacidosis without any immunological autoantibody to islet antigens of classic type 1 diabetes but demonstrate clinical and metabolic features of type 2 diabetes. Accumulating data indicated that gender-related body fat distribution, hormonal and genetic factors are associated with the diabetic process and the human glucose homeostasis and metabolism. A controversial question is whether and to what degree those factors contribute to the phenomenon of male predominance in KPDM. The present review focuses on the role of gender hormones and other potential precipitating factors in explaining the male predominance in KPDM patients.
\end{abstract}

\section{Contents}

1. Introduction

2. Body fat distribution and insulin sensitivity

3. Gender-related hormones and other hormones

4. Glucolipotoxicity susceptibility and lifestyle

5. Susceptibility to potential virus infections

6. Genetic susceptibility

7. Conclusion

Correspondence to: Dr Huiwen Tan, Department of Endocrinology and Metabolism, West China Hospital of Sichuan University, 37 Guoxue Road, Chengdu, Sichuan 610041, P.R. China

E-mail: tanhuiwen2005@163.com

Key words: diabetic ketoacidosis, ketosis-prone diabetes, male predominance, gender difference, insulin sensitivity

\section{Introduction}

Recent evidence indicates that ketosis-prone diabetes mellitus (KPDM), which was once described as atypical diabetes mellitus, idiopathetic type 1 diabetes (type 1B diabetes) or flatbush diabetes, shows a 2- or 3-fold higher prevalence in men compared to women (1-5). Previous studies by our study group and other investigators indicate that the majority of patients with KPDM are obese, young and middle-aged patients with a strong family history of type 2 diabetes (2). Of note, KPDM is mostly observed in males. Although our understanding of the pathogenesis of diabetic ketoacidosis (DKA) is increasing, it remains unknown why obese male individuals are more prone to DKA (2). The male predominance of KPDM appears to be independent of the degree of obesity and age at presentation, even though the syndrome is strongly linked to obesity $(2,3)$. Vital information is lacking to help identify the individuals at risk and to prevent or postpone the onset of DKA in patients with KPDM. Gender differences in KPDM and the role of gonadal hormones and other factors in modulating insulin sensitivity and glycometabolism are of increasing interest (6-8).

The male-to-female ratios of KPDM patients were 1.5:1 to $6: 1$ according to previous studies (2,9-12). In 1987, Winter et al (9) first systematically reported a small cohort of African-American adolescents with severe hyperglycemia and DKA, with a lack of any clear inducements. They specified this subtype of syndrome as atypical diabetes. In the past two or three decades, clinical presentations of the syndrome have been increasingly recognized worldwide. KPDM was recently reported in a non-Caucasian population, including Sub-Sahala-African or West African (3), Hispanic descent (10), Japanese (12) and Chinese populations $(13,14)$. In 2004, Mauvais-Jarvis et al (3) published a 10-year longitudinal study of KPDM in immigrants from sub-Saharan-Africa who were living in Paris, France. They reported that $>3$ quarters of the patients with KPDM were male. The following year, Maldonado et al (14) reported a large, longitudinal, prospective study of multiethnic patients with four different subforms of KPDM according to the $\beta$-cell function and antibodies of patients in Texas. The male-to-female ratio in this study was 1.72:1. In Asia, the male predominance in patients with KDPM is more evident. Yamada and Nonaka (12) reported 8 Japanese adolescents who were all obese and had a soft-drink history. The syndrome was designated as 'ketoacidosis-onset type 2 diabetes' and 'soft-drink diabetes' (10). Investigators 
Table I. Gender differences and obesity in ketosis-prone diabetes.

\begin{tabular}{lcccccccc}
\hline Parameters & Chengdu & Hong Kong & Taiwan & Tokyo & Paris & Atlanta & Houston & TX, CA and NY \\
\hline Male KPDM, & 96.7 & 72.7 & 70.0 & 100.0 & 75.6 & 63.6 & 55.6 & 75.6 \\
$\%(\mathrm{n} / \mathrm{N})$ & $(29 / 30)$ & $(8 / 11)$ & $(28 / 40)$ & $(8 / 8)$ & $(115 / 152)$ & $(49 / 77)$ & $(59 / 106)$ & $(115 / 152)$ \\
BMI, kg/m & $29.3 \pm 2.8$ & $24.9 \pm 3.9$ & 22.2 & - & $24.6 \pm 4.8$ & $37 \pm 1.0$ & $30.3 \pm 6.9$ & M: $27.9 \pm 5.6$ \\
& & & & & & & & F: $30.3 \pm 6.5$ \\
Obese, $\%$ & 70.0 & 72.7 & - & 100.0 & 75.0 & 100.0 & - & - \\
Refs.) & $(2)$ & $(13)$ & $(14)$ & $(12)$ & $(11)$ & $(21)$ & $(15)$ & $(33)$ \\
\hline
\end{tabular}

N, total number; BMI, body mass index; M, male; F, female; TX, Texas; CA, California; NY, New York.

Table II. Potential factors of diabetic ketosis in KPDM and possible mechanisms of male predominance.

Triggers of diabetic ketosis in KPDM

Infection

Non-compliance with therapy such as omission of/or inadequate insulin therapy

New onset diabetes

Stress

Surgery, heart attack and stroke

Alcohol or drug abuse
Precipitate factors may lead to male predominance in KPDM

Difference in body fat distribution

Gender-related hormones including estrogen, testosterone, SHBG and other hormones (leptin and GH)

Lifestyle including smoking, alcohol abuse and heavy soft-drink consumption

Susceptibility to glucotoxicity and/or lipotoxicity

Virus infection (HHV-8 or Coxsaekie B)

X-linked G6PD deficiency

Mutation Gly574Ser in the HNF1A gene, GCK, HNF, PDX-1, $P A X-4$ and $N G N 3$

SHBG, sex hormone-binding globin; GH, growth hormone; HHV-8, human herpes virus 8; GCK, glucokinase; $H N F$, hepatocyte nuclear factor; $P D X-1$, pancreatic and duodenal homeobox-1; $P A X-4$, paired box gene-4; NGN3, neurogenin3; G6PD, glucose-6-phosphate dehydrogenase; HNF1A, hepatocyte nuclear factor 1 homeobox A; KPDM, ketosis-prone diabetes mellitus.

in Hong Kong and Taiwan reported that 8 of $11(73 \%)$ and 28 of $40(70 \%)$ cases were male patients, respectively $(13,14)$. Consistent with prior studies regarding KPDM, a cohort of KPDM patients were characterized as male or female, and $96.7 \%$ of the individuals were male in one of our recent studies in Western China (2). The gender differences and occurrence of obesity in KPDM are shown in Table I.

The mechanisms of male predominance in KPDM are unknown. It is often assumed that there is little or no gender bias within either type 1 or type 2 diabetes mellitus. Recent studies have shown that male type 2 diabetic patients are prone to have unproved diabetic ketosis, particularly the overweight individuals. The potential triggers are complex and the underlying mechanisms of ketosis-prone in patients with KPDM are varied (Table II). The male excess in KPDM indicates that there may be other causes and mechanisms involved. In the limited discussion in the literature on the possible mechanisms underlying the excess susceptibility of males to KPDM, it has been suggested that the lifestyle and gender hormones in males may be responsible. It is our belief that it has been attributed to body fat distribution, hormonal factors, susceptibility to glucotoxicity and lipotoxicity, and changes in insulin sensitivity, which duality may result in a different and relatively severe deficiency of insulin action on glucose metabolism in KPDM.

\section{Body fat distribution and insulin sensitivity}

KPDM was more frequent in men compared to women and in obese compared to non-obese participants. Gender differences in KPDM may be associated with a different impact of obesity in men and women. An important gender difference in body fat distribution is generally observed, and consideration should be given between the genders. Gender differences in body composition and/or insulin sensitivity are evident in humans throughout the lifespan. Gender differences in body fat distribution are well-recognized, with men accumulating adipose tissue in the visceral area to a much greater extent than women. Accordingly, the amount of visceral adipose tissue fat is twice as high in men as in women (16). Otsuki et al (6) found that there were gender differences of age-dependent changes of insulin sensitivity, but not of insulin secretory activity, in Japanese non-diabetic subjects. This gender difference in body fat distribution and its associated insulin sensitivity has been shown to explain a major portion of the differing metabolic profiles and DKA risk in male and female diabetic patients.

\section{Gender-related hormones and other hormones}

There is strong evidence for gender differences in the incidence and presentation of the majority of disease processes (17), in 
addition to a long-standing knowledge that men and women differ in the most basic metabolic processes. There is a well-known sexual dimorphism in terms of androgen metabolism. Evidence showed that estrogen may have favorable effects on insulin sensitivity, whereas androgens may have undesirable effects on it. Androstenedione is known to be the precursor of testosterone and estrone. The elevated androstenedione levels during lipid/heparin infusion resulted in an increase of testosterone and dihydrotestosterone. However, a potential mechanism linking elevated free fatty acids/triglycerides and androgens was not presented in the study (19). Other hormones also influence insulin sensitivity, accounting for gender differences of the development of diabetic ketosis. For example, serum leptin concentrations have been demonstrated as higher in female mice compared to males. In addition, the effects of growth hormones on glucose, lipid and protein metabolism were also revealed recently (18). However, there are few population-based studies of gender differences in insulin sensitivity (20).

\section{Glucolipotoxicity susceptibility and lifestyle}

Increasing clinical data suggest that patients with KPDM are more susceptible to glucotoxicity or lipotoxicity, if not glucolipotoxicity, in obese male individual $(21,22)$. As it is frequently associated with obesity and hyperlipidemia, as well as hyperglycemia, certain investigators have examined whether high levels of free fatty acids or other lipids may be the underlying trigger of unprovoked DKA or ketosis. Accumulating evidence shows that severe glucotoxic blunting of an intracellular pathway leading to insulin secretion may contribute to the reversible $\beta$-cell dysfunction characteristic of KPDM patients (22). Differences in the lifestyle of males, including active use of cocaine or alcohol abuse, may also have an impact on the incidence of diabetes and these findings call for further studies in this respect (23).

\section{Susceptibility to potential virus infections}

Is it possible that the KPDM in Chinese patients described by Tan et al (13) represents an attack by disease source factors, such as virus or bacteria. However, there was no adequate evidence that patients with KPDM had an infectious illness. It has been hypothesized that differences in susceptibility to infections may be associated with an X- or Y-linked histocompatibility locus. It has been shown that gender differences with male predominance occur in the incidence of mumps, Coxsaekie B virus, adenovirus, echovirus and polio virus infections in humans (24-27). Sobngwi et al (27) found a high association between KPDM and human herpes virus- 8 infection and evidenced that this virus was able to infect human $\beta$-cells in vitro (25). The possibility of virus infections for the development of KPDM remains uncertain. Recent findings of male predominance do not allow any conclusions to be drawn concerning increased susceptibility to virus infections in males (26).

\section{Genetic susceptibility}

Recent evidence shows that genetic susceptibility contributes to KPDM. However, it remains unknown whether the model is polygenic or has a major gene influence. A point mutation, Gly574Ser, in the hepatocyte nuclear factor 1 homeobox A (HNF1A) gene was proposed as a marker of KPDM in African-American adolescents (28), but a recent study in adults excluded this association (29). Sonbgwi et al (30) investigated the possibility of X-linked glucose-6-phosphate dehydrogenase (G6PD) deficiency as a genetic basis for the male-predominant KPDM phenotype in West African populations. They found a higher prevalence of functional G6PD deficiency in the KPDM patients compared to patients with type 2 diabetes and an association between $\beta$-cell functional reserve and erythrocyte G6PD activity; however, the functional G6PD deficiency was not matched by a higher prevalence of G6PD gene mutations. Therefore, G6PD dysfunction may contribute to impaired $\beta$-cell defense against oxidant stress with regards to acute hyperglycemia, but its cause does not appear to be a genetic mutation. A previous study confirmed that the majority (70\%) of the A- $\beta$ - KPDM patients had no significant causal polymorphisms in either the proximal promoter or coding regions of 7 genes, including glucokinase, $H N F 1 A$ and $H N F 1 B$, pancreatic and duodenal homeobox $1, \beta 2 /$ NeuroD1 and paired box gene 4 . The investigators regarded that A- $\beta$ - KPDM is more likely to not be a predominantly monogenic diabetic syndrome (31,32). The previous study by Louet et al (33) showed that neurogenin-3 variations may influence the pathogenesis of KPDM in West African populations but did not show an association with gender distribution. Taken together, these results suggest that upon further consideration and following future directions, these studies have the potential to inform us with regards to gender differences in the regulation of metabolic signals and how to proceed. Additional pathophysiology insight is required to provide an improved understanding of gender distribution with male predominance in KPDM.

\section{Conclusion}

In conclusion, KPDM is a complex and heterogeneous disease with environmental and genetic factors influencing pathophysiology and clinical progression significantly (32). Currently, the syndromes of KPDM and its specific complications appear to be increasingly recognized worldwide. However, gender differences between men and women with DKA and the mechanisms of the male predominance in KPDM are too complicated and often subtle. Proper clinical epidemiological surveys remain to be conducted. Increasing information provides insights into possible differences in pathogenesis, and intervention strategies of KPDM. Gender differences in KPDM could provide important information for the pathogenesis of DKA. The male predominance in KPDM may have therapeutic indications for potential tailored pharmacological intervention in diabetes and its associated complications. Further research on this subject should be conducted.

\section{References}

1. Balasubramanyam A, Nalini R, Hampe CS and Maldonado M: Syndromes of ketosis-prone diabetes mellitus. Endocr Rev 29: 292-302, 2008

2. Tan H, Yu Y, Yu H and Wang C: The study on insulin secretion and insulin action in overweight and lean Chinese diabetic subjects with unprovoked ketosis. Diabetes 57: 22311-1717, 2008. 
3. Mauvais-Jarvis F, Sobngwi E, Porcher R, Riveline JP, Kevorkian JP, Vaisse C, Charpentier G, Guillausseau PJ, Vexiau P and Gautier JF: Ketosis-prone type 2 diabetes in patients of sub-Saharan African origin: Clinical pathophysiology and natural history of beta-cel dysfunction and insulin resistance. Diabetes 53: 645-653, 2004.

4. Smiley D, Chandra P and Umpierrez GE: Update on diagnosis, pathogenesis and management of ketosis-prone Type 2 diabetes mellitus. Diabetes Manag (Lond) 1: 589-600, 2011.

5. Zhang X, Cui X, Li F, Wang S, Liu X, Hui L, Song N and Li N Association between diabetes mellitus with metabolic syndrome and diabetic microangiopathy. Exp Ther Med 8: 1867-1873, 2014

6. Otsuki M, Kasayama S, Saito H, Mukai M and Koga M: Sex differences of age-dependent changes of insulin sensitivity in Japanese nondiabetic subjects. Diabetes Care 28: 2590-2591, 2005.

7. Sun M, Huang X, Jiang L, Yan Y, Li B, Zhong W, Chen J, Zhang Y, Wang Z, Li J, et al: Characterization of $\beta$-cell function and insulin resistance in overweight Chinese adolescents with normal glucose tolerance. Exp Ther Med 6: 547-551, 2013.

8. Galipeau DM, Yao L and McNeill JH: Relationship among hyperinsulinemia, insulin resistance, and hypertension is dependent on sex. Am J Physiol Heart Circ Physiol 283: H562-H567, 2002.

9. Winter WE, Maclaren NK, Riley WJ, Clarke DW, Kappy MS and Spillar RP: Maturity-onset diabetes of youth in black Americans. N Engl J Med 316: 285-291, 1987.

10. Piñero-Piloña A, Litonjua $\mathrm{P}$, Aviles-Santa L and Raskin P: Idiopathic type 1 diabetes in Dallas, Texas: A 5-year experience. Diabetes Care 24: 1014-1018, 2001.

11. Louet JF, Smith SB, Gautier JF, et al: Gender and neurogenin3 influence the pathogenesis of ketosis-prone diabetes. Diabetes Obes Metab 10: 912-920, 2008.

12. Yamada K and Nonaka K: Diabetic ketoacidosis in young obese Japanese men. Diabetes Care 19: 671, 1996.

13. Tan KC, Mackay IR, Zimmet PZ, Hawkins BR and Lam KS: Metabolic and immunologic features of Chinese patients with atypical diabetes mellitus. Diabetes Care 23: 335-338, 2000.

14. Hsin Yu E, Guo HR and Wu TJ: Factors associated with discontinuing insulin therapy after diabetic ketoacidosis in adult diabetic patients. Diabet Med 18: 895-899, 2001.

15. Maldonado MR, Otiniano ME, Cheema F, Rodriguez L and Balasubramanyam A: Factors associated with insulin discontinuation in subjects with ketosis-prone diabetes but preserved $\beta$-cell function. Diabet Med 22: 1744-1750, 2005.

16. Blouin K, Boivin A and Tchernof A: Androgens and body fat distribution. J Steroid Biochem Mol Biol 108: 272-280, 2008

17. van Nas A, Guhathakurta D, Wang SS, Yehya N, Horvath S, Zhang B, Ingram-Drake L, Chaudhuri G, Schadt EE, Drake TA, et al: Elucidating the role of gonadal hormones in sexually dimorphic gene coexpression networks. Endocrinology 150: 1235-1249, 2009.

18. Hursting SD and Hursting MJ: Growth Signals, Inflammation, and Vascular Perturbations Mechanistic Links Between Obesity, Metabolic Syndrome, and Cancer. Arterioscler Thromb Vasc Biol 32: 1766-1770, 2012.

19. Shi H, Strader AD, Woods SC and Seeley RJ: Sexually dimorphic responses to fat loss after caloric restriction or surgical lipectomy. Am J Physiol Endocrinol Metab 293: E316-E326, 2007.

20. Donahue RP, Prineas RJ, DeCarlo Donahue R, Bean JA and Skyler JS: The female 'insulin advantage' in a biracial cohort: Results from the Miami Community Health Study. Int J Obes Relat Metab Disord 20: 76-82, 1996.
21. Umpierrez GE, Smiley D and Kitabchi AE: Narrative review: Ketosis-prone type 2 diabetes mellitus. Ann Intern Med 144: 350-357, 2006.

22. Umpierrez GE, Smiley D, Gosmanov A and Thomason D Ketosis-prone type 2 diabetes: Effect of hyperglycemia on $\beta$-cell function and skeletal muscle insulin signaling. Endocr Pract 13 283-290, 2007.

23. Nyenwe EA, Loganathan RS, Blum S, Ezuteh DO, Erani DM, Wan JY, Palace MR and Kitabchi AE: Active use of cocaine: An independent risk factor for recurrent diabetic ketoacidosis in a city hospital. Endocr Pract 13: 22-29, 2007.

24. Ng MC, Lee SC, Ko GT, Li JK, So WY, Hashim Y, Barnett AH, Mackay IR, Critchley JA, Cockram CS, et al: Familial early-onset type 2 diabetes in Chinese patients: Obesity and genetics have more significant roles than autoimmunity. Diabetes Care 24 663-671, 2001

25. Gautier JF: Ketosis-prone type 2 diabetes mellitus in sub-Saharan Africans: Possible contribution of human herpes virus 8 infection. Med Sci (Paris) 24: 665-666, 2008 (In French).

26. Muenchhoff M and Goulder P Jr: Sex differences in pediatric infectious diseases. J Infect Dis 209 (suppl 3): S120-S126, 2014.

27. Sobngwi E, Choukem SP, Agbalika F, Blondeau B, Fetita LS, Lebbe C, Thiam D, Cattan P, Larghero J, Foufelle F, et al: Ketosis-prone type 2 diabetes mellitus and human herpesvirus 8 infection in sub-saharan africans. JAMA 299: 2770-2776, 2008.

28. Boutin P, Gresh L, Cisse A, Hara M, Bell G, Babu S, Eisenbarth G and Froguel P: Missense mutation Gly574Ser in the transcription factor HNF-1alpha is a marker of atypical diabetes mellitus in African-American children. Diabetologia 42: 380-381, 1999.

29. Mauvais-Jarvis F, Boudou P, Sobngwi E, Riveline JP, Kevorkian JP, Villette JM, Porcher R, Vexiau P and Gautier JF: The polymorphism Gly574Ser in the transcription factor HNF-1alpha is not a marker of adult-onset ketosis-prone atypical diabetes in Afro-Caribbean patients. Diabetologia 46: 728-729, 2003.

30. Sobngwi E, Gautier JF, Kevorkian JP, Villette JM, Riveline JP, Zhang S, Vexiau P, Leal SM, Vaisse C and Mauvais-Jarvis F: High prevalence of glucose-6-phosphate dehydrogenase deficiency without gene mutation suggests a novel genetic mechanism predisposing to ketosis-prone diabetes. J Clin Endocrinol Metab 90: 4446-4451, 2005.

31. Sumner AE, Farmer NM, Tulloch-Reid MK, Sebring NG, Yanovski JA, Reynolds JC, Boston RC and Premkumar A: Sex differences in visceral adipose tissue volume among African Americans. Am J Clin Nutr 76: 975-979, 2002.

32. Haaland WC, Scaduto DI, Maldonado MR, Mansouri DL, Nalini R, Iyer D, Patel S, Guthikonda A, Hampe CS, Balasubramanyam A, et al: A- $\beta$-subtype of ketosis-prone diabetes is not predominantly a monogenic diabetic syndrome. Diabetes Care 32: 873-877, 2009.

33. Louet JF, Smith SB, Gautier JF, Molokhia M, Virally ML, Kevorkian JP, Guillausseau PJ, Vexiau P, Charpentier G, German MS, et al: Gender and neurogenin3 influence the pathogenesis of ketosis-prone diabetes. Diabetes Obes Metab 10: 912-920, 2008 